\title{
Personalized Medicine in Advanced Cholangiocarcinoma
}

\author{
Anuhya Kommalapati, ${ }^{1}$ James Yu, ${ }^{2}$ and Richard Kim' \\ 1. Department of Gastrointestinal Oncology, H Lee Moffitt Cancer Center, Tampa, FL, USA; \\ 2. Department of Internal Medicine, AdventHealth Orlando, Orlando, FL, USA
}

$\mathrm{P}$

ersonalized medicine is the new-generation concept of managing cancer, which primarily focuses on the development of targeted therapies blocking specific cellular pathways that potentiate the tumorigenesis and identify people that respond best. The combination of gemcitabine and a platinum agent, remains the first-line therapy in advanced cholangiocarcinoma. Thus far, there are no specific guidelines on the next step in the care of patients who progressed on, or could not tolerate, the first-line therapy. However, a better knowledge of molecular pathogenesis and advancements in the development of targeted therapy offers hope that we may improve outcomes in advanced cholangiocarcinoma. Among the newly discovered molecular alterations, targeting isocitrate dehydrogenase (IDH1/2) mutations, fibroblast growth factor receptor 2 (FGFR2) fusions, RAS-MAPK pathway activation, BRCA1/2 mutations, NTRK fusions, and human epidermal growth factor receptor 2 (HER2), hold great promise for improving the future management of cholangiocarcinoma. In tumors with high microsatellite instabilities or mismatch repair deficiencies irrespective of programmed death ligand (PD-L) 1 expression, immunotherapy, alone or in combination with targeted agents and chemotherapy, are currently being evaluated. This review article details the potential targetable molecular pathways and future directions in implementing personalized medicine in this dismal cancer.

\section{Keywords}

Personalized medicine, cholangiocarcinoma, FGFR, IDH, BRCA

Disclosures: Richard Kim has received honoraria from Lilly, BMS, and Bayer outside the submitted work. Anuhya Kommalapati and James Yu have no financial or non-financial relationships or activities to declare in relation to this article.

Review Process: Double-blind peer review.

Compliance with Ethics: This article involves a review of literature and does not report on new clinical data, or any studies with human or animal subjects performed by any of the authors.

Authorship: All named authors meet the criteria of the International Committee of Medical Journal Editors (ICMJE) for authorship for this manuscript, take responsibility for the integrity of the work as a whole and have given final approval for the version to be published.

Access: This article is freely accessible at touchONCOLOGY.com (c) Touch Medical Media 2020.

Received: January 7, 2020

Accepted: February 7, 2020

Published Online: March 20, 2020

Citation: Oncology \& Hematology Review (US) 2020;16(1):52-8

Corresponding Author: Richard Kim,

H Lee Moffitt Cancer Center, 12902 Magnolia Drive FOB-2 Tampa, FL 33612, USA. E: richard.kim@moffitt.org

Support: No funding was received in the publication of this article.
Cholangiocarcinoma constitutes approximately $3 \%$ of all gastrointestinal malignancies, and the incidence is on the rise, especially the intrahepatic subtype. These tumors are anatomically broadly classified into intrahepatic, perihilar, and distal cholangiocarcinoma. Recent understanding of tumorigenesis pathways has shown that there exists notable differences in molecular pathogenesis between the intrahepatic versus extrahepatic versus gallbladder cancers. ${ }^{1}$ For instance, a genomic analysis of 489 cholangiocarcinoma tissue specimens revealed notable genetic heterogeneity of the tumors, which signifies the variations in molecular profile depending upon the location and etiology. In addition, a comprehensive evaluation of 1,104 cholangiocarcinoma tumor specimens showed that the most common genes (in at least 10\% of the specimens analyzed) involved in the tumorigenesis were TP53, CDKN2A/2B, KRAS, ARID1A, SMAD4, BAP1, isocitrate dehydrogenase (IDH1), PBRM, and fibroblast growth factor receptor 2 (FGFR2) (in the order of highest to lowest frequency). ${ }^{3}$ Interestingly, the study identified potentially targetable aberrations in at least $43 \%$ of the tumor samples analyzed, reinforcing the potential role of precision medicine in advanced cholangiocarcinoma.

Initially, molecular therapies targeting epidermal growth factor receptor (EGFR) and vascular endothelial growth factor receptor (VEGFR) pathways did not show encouraging results in unselected populations. For example, although EGFR protein overexpression has been implicated in 11-27\% of intrahepatic and 5-9\% of extrahepatic cholangiocarcinoma, EGFR inhibitor erlotinib showed only a modest benefit in an unselected cohort of cholangiocarcinoma. ${ }^{4}$ Similarly, cabozantinib, a mesenchymal epithelial transition (MET) inhibitor, also yielded disappointing results in a phase ॥ study involving 19 patients with cholangiocarcinoma. ${ }^{5}$ In contrast, encouraging results were seen in patients with identified mutational targets, especially in tumors harboring IDH1/2 mutations, FGFR2 fusions in intrahepatic cholangiocarcinoma, and human epidermal growth factor receptor 2 (HER2) mutations in gallbladder cancer. ${ }^{6}$ These mixed results show that a better knowledge of molecular pathogenesis and advancements in the development of targeted therapy offers hope that we may improve outcomes in a subset of patients with advanced cholangiocarcinoma. Among the newly discovered molecular alterations, targeting IDH1/2 mutations, FGFR2 fusions, RAS-MAPK pathway activation, BRCA1/2 mutations, NTRK fusions, and HER2 receptors, there is great promise for improving the future management of cholangiocarcinoma. In tumors with high microsatellite instabilities or mismatch repair deficiencies irrespective of programmed death ligand 1 (PD-L1) 
expression, immunotherapy, alone or in combination with targeted agents and chemotherapy are currently being evaluated. This article reviews the key molecular pathological pathways involved in the tumorigenesis of cholangiocarcinoma and focuses on the molecular targeted therapies acting on these pathways.

\section{Molecular pathogenesis and targeted therapies being evaluated in cholangiocarcinoma}

Molecular studies have determined the key differences in the tumorigenesis of cholangiocarcinoma are based on anatomical subtype. As such, a comprehensive transcriptome analysis and whole exome sequencing of these tumors have shown distinct genomic aberrations in intrahepatic, perihilar, and distal subtypes.? A study by Nakamura et al. showed that the aberrations implicated in the tumorigenesis of intrahepatic cholangiocarcinoma involved IDH1, IDH2, FGFR1, FGFR2, FGFR3, EPHA2, and BAP1 genes. ${ }^{\text {? }}$ On the contrary, there was a preferential involvement of AT-rich interactive domain-containing protein 1B (ARID1B), ELF3, polybromo 1 (PBRM1), protein kinase CAMP-activated catalytic subunit alpha (PRKACA), and PRKACB genes in perihilar and distal subtypes. ${ }^{7}$ This clearly shows that targeted therapies should be tailored to the specific subtype of cholangiocarcinoma, and hence the concept of personalized medicine based on the subtype of cholangiocarcinoma.

\section{Isocitrate dehydrogenase mutations}

$\mathrm{IDH}$ (isoforms 1, 2, and 3) is involved in the conversion of isocitrate to $\alpha$-ketoglutarate in the Krebs cycle. The key difference between these isoforms is that IDH isoform 1 is limited to cytoplasm whereas isoforms 2 and 3 are located in mitochondrion. Epigenetic alterations of IDH1/2 genes are implicated in about $14 \%$ of intrahepatic subtypes, which leads to increased conversion of isocitrate to D-2-hydroxyglutarate. D-2 hydroxyglutarate acts as an oncometabolite, which inhibits $\alpha$-ketoglutarate-dependent dioxygenases, increasing levels of TP53 proteins, leading to hypermethylation of DNA, potentiating tumorigenesis by altering cell differentiation. ${ }^{8,9}$

Ivosidenib (AG-120) is an oral IDH1 inhibitor which was evaluated in a dose escalation and expansion phase I trial involving 73 patients with cholangiocarcinoma who failed first-line therapy with gemcitabine and cisplatin doublet. ${ }^{10}$ Ivosidenib resulted in encouraging outcomes in terms of tolerance, and overall response rate of $62 \%$-in which $56 \%$ had stable disease and the remaining $6 \%$ had partial response-which translated to a progression-free survival of $40 \%$ in 6 months. At a molecular level, ivosidenib showed morphological alterations in IDH1-mutated cholangiocytes. ${ }^{11}$ Ivosidenib is currently being evaluated in a phase III multicenter, randomized clinical trial (ClarlDHy trial) in 185 patients with advanced intrahepatic cholangiocarcinoma harboring IDH1 mutations who failed one or two lines of chemotherapy. The trial has a primary endpoint of progression-free survival, while the secondary endpoints include drug tolerance and overall survival (OS; ClinicalTrials.gov identifier: NCT02989857). ${ }^{12}$ Interim results of the trial were presented at the European Society of Medical Oncology (ESMO) 2019 annual conference. ${ }^{13}$ The qualified patients were randomized to either ivosidenib $500 \mathrm{mg}$ or placebo. Notably, the patients enrolled on to the placebo arm who had radiological progression during the course of the study were allowed to cross over to the ivosidenib arm. Ivosidenib resulted in promising outcomes in terms of progression-free survival (median: 2.7 versus 1.4 months; hazard ratio
$[\mathrm{HR}]=0.37$; 95\% confidence interval $[\mathrm{Cl}] 0.25-0.54 ; \mathrm{p}<0.001)-32 \%$ and $22 \%$ of the ivosidenib cohort had progression-free survival at the end of 6 and 12 months, respectively.

These encouraging results translated to a disease control rate of 53\%, in which $51 \%$ had stable disease and $2 \%$ had partial response. This is in contrast to none of the patients in the placebo arm having progression-free survival at the end of 6 months, while no patients were left in placebo group at the end of 12 months. Ivosidenib showed a trend towards improvement in OS compared with that of placebo (10.8 versus 9.7 months). On exploratory analysis using the Rank-Preserving Structural Failure Time method as if the patients were never crossed over, statistically and clinically meaningful differences were seen in estimated OS (10.8 versus 6.0 months, $\mathrm{p}<0.001$ ). Ivosidenib was well-tolerated with no clinically meaningful differences in grade III side effects between the two arms (46\% versus 36\%). The phase III trial is the first of its kind that has demonstrated promising results with targeted therapy in patients with cholangiocarcinoma, which has opened doors to precision medicine in this dismal cancer.

\section{Fibroblast growth factor receptor 2 genetic fusions}

FGFR2 fusions with other genes such as BICC1, PPHLN1, MGEA5, transforming acidic coiled-coil containing protein 3 (TACC3), and coiled-coil domain-containing protein $6(C C D C 6)$ are implicated in the pathogenesis of 13-20\% of intrahepatic cholangiocarcinoma tumors. ${ }^{14,15}$ Mutations in FGFR1 and FGFR3 have also been encountered in the carcinogenesis of intrahepatic subtype.? In addition, upregulation of FGFR 1, 2, and 4 were demonstrated in the cholangiocarcinoma cell lines, which was thought to be mediated by a transcriptional cofactor, yes-activated protein. ${ }^{16}$ These fusions are thought to activate the downstream signaling pathways of RAS-MAPK and JAK-STAT. ${ }^{17-19}$ Furthermore, FGF ligand has been implicated in the MEK1/2 phosphorylation contributing to migration of cholangiocarcinoma cells. ${ }^{20}$ Some FGFR2 fusions such as FGFR2-AHCYL1, and FGFR2-KIAA158 were associated with KRAS mutations. 17,21 In addition, BAP1, TP53, and CDKN2A/B mutations were the most common co-existing mutations. While co-existing BAP1 mutations do not influence median OS, co-existing mutations with TP53 and CDKN2A/2B are associated with inferior median OS $(p=0.04) .^{22}$ Notably, FGFR2 fusions were shown to be associated with female sex (13\% versus $4 \%$ ), younger age at diagnosis (52 versus 65 years) and better median OS (123 versus 37 months). ${ }^{15}$

FGFRs are known to harbor tyrosine kinase domains, as such, tyrosine kinase inhibitors targeting FGFRs have been extensively evaluated in intrahepatic cholangiocarcinoma. ${ }^{23}$ In a phase II multicenter clinical trial, infigratinib (BGJ398) showed promising outcomes in 61 patients with advanced cholangiocarcinoma that failed first-line gemcitabine-based chemotherapy. ${ }^{24}$ Among these 61 patients enrolled, 48 patients were shown to harbor FGFR2 fusions, eight and three patients had mutations and amplifications of FGFR2, respectively. The primary endpoint of the study was to evaluate the overall response rate, which was accounted to be $14.8 \%$ in the entire cohort, while the patients harboring FGFR2 fusions had an overall response rate of $18.8 \%$. Infigratinib resulted in a disease-control rate of $75.4 \%$, which translated to a median progression-free survival of 5.8 months. While the drug was well tolerated, the most common side effects noted were hyperphosphatemia, fatigue, stomatitis, and alopecia primarily resulting from blocking other FGFR pathways. 
The authors presented (at the ESMO 2018 annual meeting) updated data of the same trial that enrolled 71 patients with advanced intrahepatic cholangiocarcinoma and with FGFR2 fusions, which showed partial response, stable disease, and progressive disease in $24 \%, 58 \%$, and $11 \%$, respectively. ${ }^{25}$ One has to be cautiously optimistic with these FGFR-targeting tyrosine kinase inhibitors given the development of resistance mechanisms, which may be attributed to the drug-binding site kinase mutations ( $\mathrm{pN549H}$ pL617V, pE565A, pK641R, pN549K, pV564F, and pK659M) and downstream pathway, PTEN/PI3K alterations. Cellular studies have shown that ponatinib and dovitinib, non-selective tyrosine kinase inhibitors, have promising activity at these point-mutations (pN540 and p549K). ${ }^{26}$

Derazantinib (ARQ-087) is another oral pan-FGFR tyrosine kinase inhibitor that has been evaluated in cholangiocarcinoma in phase I and II trials. In a phase I/II trial involving 29 patients with cholangiocarcinoma, tumor burden reduction of $10-29 \%$ was seen in three out of 12 patients with FGFR2 fusions. ${ }^{27}$ Among the 12 patients with FGFR2 fusions, six patients had a disease control for $>4$ months. The study had an expansion phase that enrolled a total of 29 patients with advanced cholangiocarcinoma that harbored FGFR2 fusions and an objective response rate of $21 \%{ }^{28} \mathrm{~A}$ single-arm phase III trial is currently enrolling patients with FGFR2 fusion-positive advanced cholangiocarcinoma that failed one or more lines of systemic therapy agents (FIGURE trial; ClinicalTrials.gov identifier: NCT03230318).

Futibatinib (TAS-120) is a covalent and irreversible inhibitor of FGFRs currently being evaluated in advanced cholangiocarcinoma. The drug was evaluated in 19 patients with cholangiocarcinoma (17 of intrahepatic subtype and two were of extrahepatic) harboring FGF/FGFR aberrations, and half of the cohort had partial response. In addition, among three patients with FGFR2 fusions who received a prior therapy with another FGFR inhibitor, one patient was seen to have partial response and another stable disease. ${ }^{29}$ Most recent updates on the same study demonstrated a tumor shrinkage in $71 \%$, with an objective response of $25 \%$ in tumors harboring FGFR2 fusions..$^{30}$ Given the irreversible binding nature of the drug to FGFR, it is currently being evaluated in the patients who had progressive disease with other FGFR inhibitors (ClinicalTrials.gov identifier: NCT02052778).

Pemigatinib is a potent FGFR inhibitor with a selective activity against FGFR 1,2 , and 3 kinases. Pemigatinib showed a promising overall response rate in patients with advanced cholangiocarcinoma harboring FGFR2 fusions or rearrangements. Recent data from the FIGHT-202 trial showed that pemigatinib resulted in an overall response rate of $35.5 \%$, with a disease control rate of $80 \%$ in 107 patients with advanced cholangiocarcinoma harboring FGFR2 fusions or rearrangements. ${ }^{31}$ On the contrary, no clinically meaningful responses were seen in other patients with FGFR gene alterations or other genetic alterations. Given these encouraging results, the United States Food and Drug Administration (FDA) accepted the new drug application of pemigatinib in FGFR2 fusion/rearrangement harboring cholangiocarcinoma for a priority review.

Table 1 summarizes key clinical trials evaluating FGFR inhibitors - infigratinib, derazantinib, erdafitinib, and futibatinib-in patients with advanced cholangiocarcinoma. ${ }^{25,28,30-33}$ Other tyrosine kinase inhibitors being evaluated in clinical trials include, but are not limited to, ponatinib
(ClinicalTrials.gov identifiers: NCT02265341, NCT02272998), erdafitinib (ClinicalTrials.gov identifier: NCT02699606), E7090 (FGFR 1, 2 and 3 receptor inhibitor), and rogaratinib (ClinicalTrials.gov identifier: NCT01976741). ${ }^{21,34}$

\section{Multi-kinase tyrosine inhibitors blocking HGF/C-MET signaling, EGFR, and MAPK pathways}

MET overexpression is implicated in the tumorigenesis of the intrahepatic subtype and perihilar/distal subtypes due to its role in angiogenesis and cell migration. ${ }^{35,36}$ In addition, C-MET is known to activate the downstream signaling pathways-JAK-STAT, AKT, and MAPK pathways. ${ }^{37}$ Similarly, EGFR has also been implicated in cell migration and angiogenesis. ${ }^{15}$ EGFR overexpression has been implicated in the carcinogenesis of cholangiocarcinoma with the highest level of expression observed in the intrahepatic subtype (38-100\%). ${ }^{31}$ Aberrant phosphorylation and overexpression of EGFR activates MAPK/ERK and p38 pathways triggering tumor growth and inhibition of apoptosis, thereby promoting the tumorigenesis. ${ }^{32}$ In intrahepatic cholangiocarcinoma, MET amplification was shown to be associated with short OS and $>5 \mathrm{~cm}$ tumor size. ${ }^{38}$

Erlotinib, a tyrosine kinase inhibitor targeting the EGFR pathway was evaluated in 268 patients with advanced cholangiocarcinoma in combination with gemcitabine and oxaliplatin doublet in a phase III trial. Though there has been an objective response in a greater percentage of patients who received erlotinib, the objective response did not translate to progression-free survival benefit. ${ }^{39}$ Similar unsatisfactory results in terms of progression-free and median os were seen with the addition of EGFR antibody, cetuximab. ${ }^{40}$ On the contrary, addition of panitumumab, another monoclonal antibody targeting EGFR, to the combination regimen of irinotecan and gemcitabine, resulted in $74 \%$ disease control rate. Moreover, the addition of these agents to chemotherapy in the presence of wild-type KRAS mutations was demonstrated to be not so effective in terms of efficacy. ${ }^{41}$ While anti-EGFR therapy with panitumumab and cetuximab yielded mixed results, ${ }^{40-42}$ the use of the c-MET inhibitor cabozantinib showed limited activity at the expense of considerable toxicity in non-biomarker driven studies. ${ }^{5}$ Hence, given the inconsistent results with EGFR and C-MET inhibitors, the role of these agents is yet to be evaluated in larger cohorts. Regorafenib, a multi-kinase inhibitor was evaluated in phase I/II trials in patients with advanced cholangiocarcinoma with modest results in terms of overall and progression-free survival (Table 2).5.39-51 Sunitinib demonstrated a progression-free survival benefit of 1.7 months in a single-arm phase II trial, ${ }^{46}$ whereas sorafenib did not yield encouraging results in various studies as detailed in Table $2 .^{47-51}$

On the contrary, a combination of BRAF inhibitor, dabrafenib and MEK inhibitor, trametinib has shown encouraging efficacy results in 33 patients with BRAFrooo mutation-positive advanced cholangiocarcinoma. ${ }^{52}$ This combination regimen resulted in a duration of response $>6$ months in $54 \%$ of the study cohort, translating to a median OS of 11.2 months. These encouraging results opened doors to the possible role of BRAF inhibitors in $B R A F^{V 600 E}$ mutation-positive advanced cholangiocarcinoma.

\section{HER2/neu (ERBB2) and HER3 receptor tyrosine kinase pathway signaling}

HER2 overexpression has been implicated in the pathogenesis of 10-16\%, $5-9 \%$, and $1 \%$ of gallbladder cancers, extrahepatic, and intrahepatic 
Table 1: Key clinical trials of selective small molecule tyrosine kinase inhibitors targeting the FGFR pathway

\begin{tabular}{|c|c|c|c|c|c|c|c|c|c|}
\hline \multirow{2}{*}{$\begin{array}{l}\text { Targeted therapy } \\
\text { agents }\end{array}$} & \multirow[t]{2}{*}{ Mechanism of therapy } & \multirow[t]{2}{*}{ Study } & \multirow[t]{2}{*}{ Phase } & \multicolumn{6}{|c|}{ Outcome } \\
\hline & & & & CR & PR & SD & ORR & $\begin{array}{l}\text { PFS } \\
\text { (months) }\end{array}$ & $\begin{array}{l}\text { OS } \\
\text { (months) }\end{array}$ \\
\hline $\begin{array}{l}\text { BGJ398 } \\
\text { (infigratinib) }\end{array}$ & $\begin{array}{l}\text { Potent FGFR1-3 inhibitor, } \\
\text { with lower FGFR4 potency }\end{array}$ & $\begin{array}{l}\text { Javle et al. }{ }^{25} \\
\text { ( } \mathrm{n}=71 \text { FGFR fusions) }\end{array}$ & $\begin{array}{l}\text { Phase II, single-arm, } \\
\text { open-label, multicenter }\end{array}$ & $0 \%$ & $31 \%$ & $53 \%$ & $31 \%$ & 6.8 & NR \\
\hline $\begin{array}{l}\text { ARQ } 087 \\
\text { (derazantinib) }\end{array}$ & $\begin{array}{l}\text { ATP-competitive } \\
\text { TKI, exhibits potent } \\
\text { activity against } \\
\text { FGFR1-3 }\end{array}$ & $\begin{array}{l}\text { Mazzaferro } \\
\text { et al. }{ }^{28}(n=29 \text { FGFR fusions) }\end{array}$ & $\begin{array}{l}\text { Phase I/II, single-arm, } \\
\text { open-label, multicenter }\end{array}$ & $0 \%$ & $21 \%$ & $62 \%$ & $21 \%$ & 5.7 & NR \\
\hline \multirow[t]{2}{*}{$\begin{array}{l}\text { JNJ-42756493 } \\
\text { (erdafitinib) }\end{array}$} & \multirow[t]{2}{*}{$\begin{array}{l}\text { Pan-FGFR TKI, } \\
\text { reversible }\end{array}$} & $\begin{array}{l}\text { Soria et al. }{ }^{32} \\
(n=11)\end{array}$ & $\begin{array}{l}\text { Phase I, single-arm, } \\
\text { multicenter }\end{array}$ & $0 \%$ & $27 \%$ & $27 \%$ & $27 \%$ & 5.1 & NR \\
\hline & & $\begin{array}{l}\text { Park et al. }{ }^{33} \\
\text { ( } \mathrm{n}=12 \text { with FGFR } \\
\text { aberrations including } \\
\text { fusions and mutations) }\end{array}$ & $\begin{array}{l}\text { Phase Ila, single-arm, } \\
\text { open-label, multicenter }\end{array}$ & $0 \%$ & $50 \%$ & $33 \%$ & $50 \%$ & 5.6 & NR \\
\hline $\begin{array}{l}\text { TAS-120 } \\
\text { (futibatinib) }\end{array}$ & $\begin{array}{l}\text { Pan-FGFR, } \\
\text { irreversible }\end{array}$ & $\begin{array}{l}\text { Meric-Bernstam et al. }{ }^{30} \\
\text { ( } \mathrm{n}=28 \text { with FGFR fusions) }\end{array}$ & $\begin{array}{l}\text { Phase I, single-arm, } \\
\text { multicenter }\end{array}$ & $0 \%$ & $25 \%$ & $53 \%$ & $25 \%$ & NR & NR \\
\hline $\begin{array}{l}\text { INCB054828 } \\
\text { (pemigatinib) }\end{array}$ & FGFR1-4 inhibitor & $\begin{array}{l}\text { Vogel et al. }{ }^{31} \\
\text { (n=107 with FGFR fusions) } \\
\text { FIGHT-202 }\end{array}$ & $\begin{array}{l}\text { Phase II, open-label, } \\
\text { single-arm }\end{array}$ & $3 \%$ & $33 \%$ & $46 \%$ & $36 \%$ & 6.9 & 21.1 \\
\hline
\end{tabular}

$A T P=$ adenosine triphosphate; $C R=$ complete response; $F G F R=$ fibroblast growth factor receptor; $N R=$ not reported; $O R R=$ overall response rate; $O S=$ overall survival; $P F S=$ progression-free survival; $P R=$ partial response; $S D=$ stable disease; $T K I=$ tyrosine kinase inhibitor .

cholangiocarcinoma, respectively.14 HER2 amplifications trigger the HER2/neu receptors thereby activating the downstream signaling pathways RAS-RAF-MEK-ERK or PI3K-AKT-MTOR resulting in the proliferation of tumor cells. Despite the presence of HER2 amplifications in a small percentage of patients with advanced cholangiocarcinoma, preliminary data in the form of a case series are encouraging. ${ }^{53}$ This case series evaluated the role of HER2-targeted therapy with trastuzumab, lapatinib, and pertuzumab in five patients with advanced cholangiocarcinoma and nine with gallbladder cancer. Though the patients with advanced cholangiocarcinoma did not show any radiological response, one, four, and three patients with gallbladder cancer had a complete response, partial response, and stable disease, respectively. Mixed responses were seen with lapatinib in a patient who harbored Val777Leu mutation in the HER2 kinase domain. While the preliminary data on HER2-targeted therapy looks promising, more detailed information and/or more robust data through clinical trials is much awaited (ClinicalTrials.gov identifiers: NCT00101036, NCT02836847).

\section{Immunotherapy}

Immunotherapy with programmed cell death-1 (PD-1), PD-L1, and cytotoxic T-lymphocyte-associated protein 4 (CTLA-4) inhibitors has emerged as a promising therapeutic option in various cancers such as melanoma, non-small cell carcinoma, and renal cell carcinoma, to name a few. In a comprehensive genomic analysis of 3,364 cholangiocarcinoma tumor specimens, $3.5 \%$ of the tumors showed $>10$ mutations/megabase (Mb), whereas $1.4 \%$ showed $>20$ mutations/Mb.54 In this analysis, PD-L1 expression was seen in 9\% of the samples analyzed, substantiating the possible role of checkpoint inhibitors. Patients with advanced cholangiocarcinoma with mismatch repair deficiency, high micro-satellite instabilities, with or without PD-L1 expression should be encouraged to participate in clinical trials evaluating the role of these agents.
Immune checkpoint inhibitors including nivolumab, pembrolizumab, and durvalumab (alone or in combination with tremelimumab) were evaluated in phase $\mathrm{I} / \mathrm{II}$ trials in patients with advanced cholangiocarcinoma. ${ }^{55-58}$ The preliminary data showed the role of checkpoint inhibitors in advanced cholangiocarcinoma. For instance, nivolumab resulted in 6- and 12-month OS rates of $71.4 \%$ and $52.3 \%$, respectively in 45 patients with advanced cholangiocarcinoma that progressed on at least one-line of systemic chemotherapy (44\% failed two lines). ${ }^{57}$ Furthermore, response rates were up to $22 \%$ with promising OS of 14 months in refractory in patients with microsatellite-stable advanced bile duct tumors. However, the results could not be duplicated with other single-agent checkpoint inhibitor studies, which are outlined in Table $3^{55-58}$ The response rate for other checkpoint inhibitors were around 10\%, irrespective of PD-L1 positivity. ${ }^{56}$

However, nivolumab in combination with systemic chemotherapy of gemcitabine and cisplatin in a Japanese cohort of 30 patients with advanced cholangiocarcinoma, seemed to demonstrate promising results. ${ }^{58}$ While nivolumab monotherapy resulted in a modest response in terms of objective response, the combination of nivolumab with gemcitabine and cisplatin resulted in a median os of 15.4 months and objective response of $37 \%$ (11 out of 30 patients). Table 3 summarizes key clinical studies with immunotherapy agents in advanced cholangiocarcinoma. The major challenge is finding a better biomarker that can predict a promising response to immunotherapy.

\section{Other potential druggable pathways and targeted therapies in advanced cholangiocarcinoma}

In addition to the above detailed genetic aberrations and pathways, BRCA1/2 mutations have been implicated in a small percentage of cholangiocarcinoma tumors. ${ }^{59}$ Molecular profiling of 1,288 
Table 2: Key clinical trials of selective multi tyrosine kinase inhibitors

\begin{tabular}{|c|c|c|c|c|c|c|c|c|c|c|}
\hline \multirow{2}{*}{$\begin{array}{l}\text { Targeted } \\
\text { therapy agents }\end{array}$} & \multirow[t]{2}{*}{ Study } & \multirow[t]{2}{*}{ Phase } & \multirow{2}{*}{\multicolumn{2}{|c|}{ Patient group }} & \multicolumn{6}{|c|}{ Outcome } \\
\hline & & & & & \multirow{2}{*}{$\begin{array}{l}\mathrm{CR} \\
0 \%\end{array}$} & \multirow{2}{*}{$\begin{array}{l}\mathrm{PR} \\
30 \%\end{array}$} & \multirow{2}{*}{$\begin{array}{l}\text { SD } \\
36 \%\end{array}$} & \multirow{2}{*}{$\begin{array}{l}\text { ORR } \\
30 \%\end{array}$} & \multirow{2}{*}{$\begin{array}{l}\text { PFS } \\
5.8 \mathrm{mo}\end{array}$} & \multirow{2}{*}{$\begin{array}{l}\text { OS } \\
9.5 \mathrm{mo}\end{array}$} \\
\hline Erlotinib & Lee et al. ${ }^{39}$ & Phase III, randomized, & First-line trial & GEMOX + erlotinib & & & & & & \\
\hline & $(n=268)$ & open-label, multicenter & & GEMOX & $2 \%$ & $14 \%$ & $51 \%$ & $16 \%$ & $4.2 \mathrm{mo}$ & $9.5 \mathrm{mo}$ \\
\hline \multirow[t]{2}{*}{ Cetuximab } & \multirow[t]{2}{*}{$\begin{array}{l}\text { Malka et al. }{ }^{40} \\
(n=150)\end{array}$} & \multirow[t]{2}{*}{$\begin{array}{l}\text { Phase II, randomized, } \\
\text { open-label, multicenter }\end{array}$} & \multirow[t]{2}{*}{ First-line trial } & $\begin{array}{l}\text { GEMOX + } \\
\text { cetuximab }\end{array}$ & $1 \%$ & $22 \%$ & $58 \%$ & $24 \%$ & $6.1 \mathrm{mo}$ & $11.0 \mathrm{mo}$ \\
\hline & & & & GEMOX & $3 \%$ & $20 \%$ & $42 \%$ & $23 \%$ & $5.5 \mathrm{mo}$ & $12.4 \mathrm{mo}$ \\
\hline \multirow[t]{3}{*}{ Panitumumab } & $\begin{array}{l}\text { Sohal et al. }{ }^{42} \\
(n=35)\end{array}$ & $\begin{array}{l}\text { Phase II, single-arm, } \\
\text { open-label }\end{array}$ & \multicolumn{2}{|l|}{ First-line trial } & $6 \%$ & $26 \%$ & $42 \%$ & $31 \%$ & $9.7 \mathrm{mo}$ & $12.9 \mathrm{mo}$ \\
\hline & \multirow[t]{2}{*}{$\begin{array}{l}\text { Leone et al. }{ }^{41} \\
(n=89)\end{array}$} & \multirow[t]{2}{*}{$\begin{array}{l}\text { Phase II, randomized, } \\
\text { open-label, multicenter }\end{array}$} & \multirow[t]{2}{*}{ First-line trial } & $\begin{array}{l}\text { GEMOX + } \\
\text { panitumumab }\end{array}$ & $2 \%$ & $24 \%$ & $49 \%$ & $27 \%$ & $5.3 \mathrm{mo}$ & $9.9 \mathrm{mo}$ \\
\hline & & & & GEMOX & $2 \%$ & $16 \%$ & $50 \%$ & $18 \%$ & $4.4 \mathrm{mo}$ & $10.2 \mathrm{mo}$ \\
\hline Cabozantinib & $\begin{array}{l}\text { Goyal et al. }{ }^{5} \\
(n=19)\end{array}$ & Phase II, single-arm & \multicolumn{2}{|c|}{ Failed at least one line of therapy } & $0 \%$ & $0 \%$ & $26 \%$ & $0 \%$ & $1.8 \mathrm{mo}$ & $5.2 \mathrm{mo}$ \\
\hline Regorafenib & $\begin{array}{l}\text { Kim et al. }{ }^{43} \\
(n=39)\end{array}$ & $\begin{array}{l}\text { Phase II, single-arm, } \\
\text { multicenter }\end{array}$ & \multicolumn{2}{|c|}{ Failed at least one line of therapy } & $0 \%$ & $6 \%$ & $56 \%$ & $6 \%$ & $3.7 \mathrm{mo}$ & $9.9 \mathrm{mo}$ \\
\hline \multirow[t]{3}{*}{ Regorafenib } & \multirow{2}{*}{$\begin{array}{l}\text { Demols et al. }{ }^{44} \\
(n=66)\end{array}$} & \multirow{2}{*}{$\begin{array}{l}\text { Phase II, randomized, } \\
\text { double-blind, } \\
\text { multicenter }\end{array}$} & \multirow{2}{*}{$\begin{array}{l}\text { Failed at least } \\
\text { one line of } \\
\text { therapy }\end{array}$} & Regorafenib & $0 \%$ & $0 \%$ & $70 \%$ & $0 \%$ & $3.0 \mathrm{mo}$ & $5.3 \mathrm{mo}$ \\
\hline & & & & Placebo & $0 \%$ & $0 \%$ & $33 \%$ & $0 \%$ & $1.5 \mathrm{mo}$ & $5.0 \mathrm{mo}$ \\
\hline & $\begin{array}{l}\text { Sun et al. }{ }^{.5} \\
(n=43)\end{array}$ & Phase $\|$, single-arm & \multicolumn{2}{|c|}{ Failed at least one line of therapy } & $0 \%$ & $11 \%$ & $44 \%$ & $11 \%$ & 15.6 wks & 31.8 wks \\
\hline \multirow[t]{5}{*}{ Sorafenib } & $\begin{array}{l}\text { Lee et al. }{ }^{47} \\
(n=39)\end{array}$ & $\begin{array}{l}\text { Phase II, single-arm, } \\
\text { open-label, } \\
\text { single-center }\end{array}$ & \multicolumn{2}{|l|}{ First-line trial } & $0 \%$ & $12 \%$ & $76 \%$ & $12 \%$ & $6.5 \mathrm{mo}$ & $14.4 \mathrm{mo}$ \\
\hline & \multirow{2}{*}{$\begin{array}{l}\text { Moehler et al. }{ }^{48} \\
(n=102)\end{array}$} & \multirow{2}{*}{$\begin{array}{l}\text { Phase II, randomised, } \\
\text { double-blind, } \\
\text { multicenter }\end{array}$} & \multirow[t]{2}{*}{ First-line trial } & GEM + sorafenib & $0 \%$ & $14 \%$ & $72 \%$ & $14 \%$ & $3.0 \mathrm{mo}$ & $8.4 \mathrm{mo}$ \\
\hline & & & & GEM & $0 \%$ & $10 \%$ & $80 \%$ & $10 \%$ & $4.9 \mathrm{mo}$ & $11.2 \mathrm{mo}$ \\
\hline & $\begin{array}{l}\text { Bengala et al. }{ }^{49} \\
(n=46)\end{array}$ & $\begin{array}{l}\text { Phase II, single-arm, } \\
\text { open-label, multicenter }\end{array}$ & \multicolumn{2}{|c|}{$\begin{array}{l}\text { 43\% first-line trial; } \\
\text { 13\% failed at one line of therapy; } \\
43 \% \text { failed at least two lines of therapy }\end{array}$} & $0 \%$ & $2 \%$ & $30 \%$ & $2 \%$ & $2.3 \mathrm{mo}$ & $4.4 \mathrm{mo}$ \\
\hline & $\begin{array}{l}\text { El-Khoueiry et al. }{ }^{50} \\
(n=36)\end{array}$ & $\begin{array}{l}\text { Phase II, single-arm, } \\
\text { swOG } 0514\end{array}$ & First-line trial & & $0 \%$ & $\begin{array}{l}6 \%, \\
\text { unconfirmed }\end{array}$ & $32 \%$ & $6 \%$ & $3.0 \mathrm{mo}$ & $9.0 \mathrm{mo}$ \\
\hline $\begin{array}{l}\text { Sorafenib + } \\
\text { erlotinib }\end{array}$ & $\begin{array}{l}\text { El-Khoueiry et al. }{ }^{51} \\
(n=34)\end{array}$ & $\begin{array}{l}\text { Phase II, single-arm, } \\
\text { swOG } 0941\end{array}$ & First-line trial & & NR & $\begin{array}{l}6 \%, \\
\text { unconfirmed }\end{array}$ & $29 \%$ & $6 \%$ & $2.0 \mathrm{mo}$ & $6.0 \mathrm{mo}$ \\
\hline Sunitinib & $\begin{array}{l}\text { Yi et al. }{ }^{46} \\
(n=56)\end{array}$ & $\begin{array}{l}\text { Phase II, } \\
\text { single-arm, } \\
\text { prospective, } \\
\text { multicenter }\end{array}$ & Second-line tri & & $0 \%$ & $9 \%$ & $41 \%$ & $9 \%$ & NR & $4.8 \mathrm{mo}$ \\
\hline
\end{tabular}

$C R=$ complete response; $G E M=$ gemcitabine; $G E M O X=$ gemcitabine and oxaliplatin; $m o=$ months; $N R=$ not reported; $O R R=$ overall response rate; $O S=$ overall survival; $P F S=$ progression-free survival; $P R=$ partial response; $S D=$ stable disease; $w K S=$ weeks.

tumor specimens of cholangiocarcinoma showed that $3 \%$ of the specimens had BRCA2 mutations while $0.6 \%$ had BRCA1 mutations. Poly-ADP ribose polymerase (PARP) inhibitors have shown promising results in other BRCA-mutated malignancies such as ovarian and breast cancers. ${ }^{60}$ Notably, on a retrospective analysis of 18 patients with BRCA-associated advanced cholangiocarcinoma, PARP inhibitors showed promising efficacious outcomes compared with standard first-line platinum-based chemotherapy. ${ }^{61}$ Several clinical trials (ClinicalTrials.gov identifiers: NCT04042831, NCT03639935, NCT03212274) are currently enrolling patients with BRCA-associated advanced cholangiocarcinoma to study the efficacy and safety of PARP inhibitors in this specific subgroup.

NTRK fusions have also been implicated in the pathogenesis of cholangiocarcinoma via activation of downstream MAPK signaling pathway.62 NTRK inhibitors, entrectinib and larotrectinib, are approved by the FDA for use in patients with tumors harboring NTRK fusions who have failed standard first- and second-line therapies. These inhibitors are currently being evaluated in advanced cholangiocarcinoma clinical trials (ClinicalTrials.gov identifiers: NCT02568267, NCT02576431). 
Table 3: Key clinical trials of immunotherapy

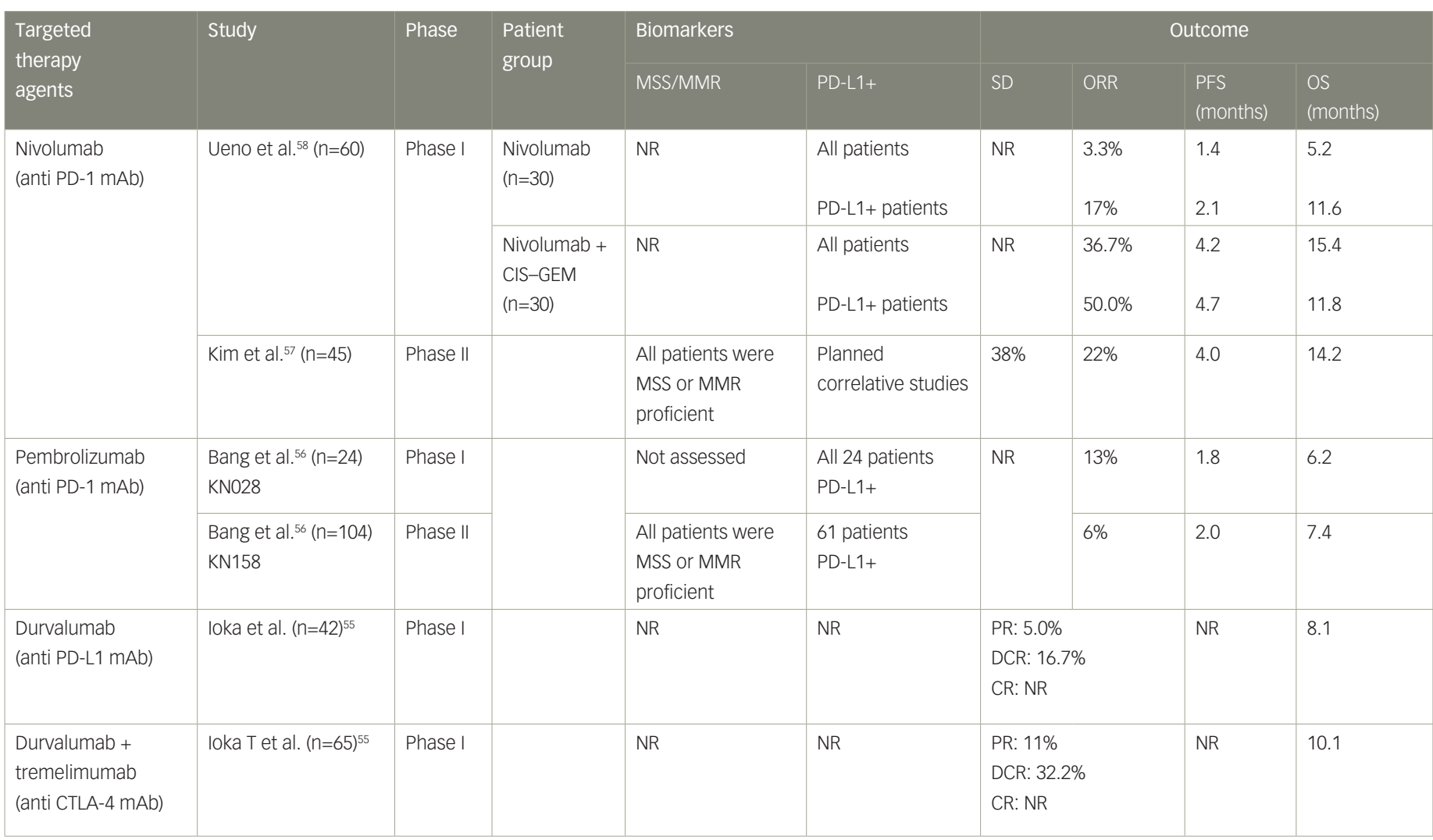

CIS-GEM = cisplatin and gemcitabine; $C R=$ complete response; $C T L A-4=$ cytotoxic T-lymphocyte-associated protein 4; $D C R=$ disease control rate; $m A b=$ monoclonal antibody; $M M R=$ mismatch repair; $M S I=$ microsatellite instabilities; $M S S=$ microsatellite stable; $N R=$ not reported; $O R R=$ overall response rate; $O S=$ overall survival; $P D-1=$ programmed cell death protein 1; PD-L1 = programmed death ligand 1; PFS = progression-free survival; $P R=$ partial response; $S D=$ stable disease.

\section{Conclusion}

As our knowledge around the tumorigenesis of cholangiocarcinoma progressed and we welcomed the advent of personalized medicine in the field of oncology, many targeted therapies have begun to be evaluated in this rare but dismal cancer. As detailed above, a considerable number of studies have shown encouraging results. Agents targeting FGFR2 fusions, IDH1/2 mutations, HER2 amplifications, BRAF mutations, and NTRK fusions have been particularly encouraging. The promising efficacy data from the ClarlDHy trial clearly showed that precision and personalized medicine is indeed applicable to advanced cholangiocarcinoma. ${ }^{12}$ Given these encouraging results, it is reasonable to recommend mutation testing for FGFR2 fusions, IDH1/2 mutations, HER2 amplifications, BRAF mutations, and NTRK fusions in patients, and they suggest that we should encourage participation in respective clinical trials. For optimal outcomes, clinical trials should be tailored to tumor biomarkers and mutations. We conclude, with optimism, that we will reach the point where we use personalized medicine as a standard of care in advanced cholangiocarcinoma. To make this dream come true we need extensive collaborative efforts to enroll patients in global clinical trials. $\square$
1. Saha SK, Zhu AX, Fuchs CS, Brooks GA. Forty-year trends in cholangiocarcinoma incidence in the U.S.: intrahepatic disease on the rise. Oncologist. 2016;21:594-9.

2. Jusakul A, Cutcutache I, Yong CH, et al. Whole-genome and epigenomic landscapes of etiologically distinct subtypes of cholangiocarcinoma. Cancer Discov. 2017;7:1116-35.

3. Silverman IM, Murugesan K, Lihou CF, et al. Comprehensive genomic profiling in FIGHT-202 reveals the landscape of actionable alterations in advanced cholangiocarcinoma. J Clin Oncol. 2019:37(Supp .15):4080.

4. Philip PA, Mahoney MR, Allmer C, et al. Phase II study of erlotinib in patients with advanced biliary cancer. J Clin Oncol. 2006;24:3069-74

5. Goyal L, Zheng H, Yurgelun MB, et al. A phase 2 and biomarker study of cabozantinib in patients with advanced cholangiocarcinoma. Cancer. 2017;123:1979-88.

6. Mahipal A, Kommalapati A, Tella SH, et al. Novel targeted treatment options for advanced

targeted treatment options for advanced

7. Nakamura H, Arai Y, Totoki Y, et al. Genomic spectra of biliary tract cancer. Nat Genet. 2015;47:1003-10.

8. Wang P, Dong Q, Zhang C, et al. Mutations in isocitrate dehydrogenase 1 and 2 occur frequently in intrahepatic cholangiocarcinomas and share hypermethylation targets with glioblastomas. Oncogene. 2013:32:3091-100.

9. Saha SK, Parachoniak CA, Ghanta KS, et al. Corrigendum: mutant IDH inhibits HNF-4alpha to block hepatocyte differentiation and promote biliary cancer. Nature. 2015;528:152.

10. Lowery MA, Abou-Alfa GK, Burris HA et al. Phase I study of AG-120, an IDH1 mutant enzyme inhibitor: Results from the cholangiocarcinoma dose escalation and expansion cohorts. J Clin Oncol. 2017:35(Suppl. 15):4015.

11. Ishii Y, Sigel C, Lowery MA, et al. AG-120 (ivosidenib), a first-in-class mutant IDH1 inhibitor, promotes morphologic changes and upregulates liver-specific genes in IDH1 mutant cholangiocarcinoma. Mol Cancer Ther. 2018;17(Suppl. 1):A071

12. Lowery MA, Abou-Alfa GK, Valle JW, et al. ClarIDHy: A phase 3 , multicenter, randomized, double-blind study of AG-120 vs placebo in patients with an advanced cholangiocarcinoma with an IDH1 mutation. J Clin Oncol. 2017:35(Suppl. 15):TPS4142.

13. Abou-Alfa GK, Macarulla Mercade $T$, Javle $M$, et al.

ClarlDHy: A global, phase III, randomized, double-blind study of ivosidenib (IVO) vs placebo in patients with advanced cholangiocarcinoma (CC) with an isocitrate dehydrogenase 1
(IDH1) mutation. Ann Oncol. 2019:30(Suppl. 5) :v872-3.

14. DeLeon $\Pi$, Ahn DH, Bogenberger JM, et al. Novel targeted therapy strategies for biliary tract cancers and hepatocellular carcinoma. Future Oncol. 2018;14:553-66.

15. Graham RP, Barr Fritcher EG, Pestova E, et al. Fibroblast growth factor receptor 2 translocations in intrahepatic cholangiocarcinoma. Hum Pathol. 2014;45:1630-8.

16. Rizvi S, Yamada D, Hirsova P, et al. A hippo and fibroblast growth factor receptor autocrine pathway in cholangiocarcinoma. J Biol Chem. 2016:291:8031-47.

17. Sia D, Losic B, Moeini A, et al. Massive parallel sequencing uncovers actionable FGFR2-PPHLN1 fusion and ARAF mutations in intrahepatic cholangiocarcinoma. Nat Commun. 2015;6:6087.

18. Arai $Y$, Totoki $Y$, Hosoda F, et al. Fibroblast growth factor receptor 2 tyrosine kinase fusions define a unique molecular subtype of cholangiocarcinoma. Hepatology. 2014;59:1427-34.

19. Moeini A, Sia D, Bardeesy N, et al. Molecular pathogenesis and targeted therapies for intrahepatic cholangiocarcinoma. Clin Cancer Res. 2016;22:291-300.

20. Narong S, Leelawat K. Basic fibroblast growth factor induces cholangiocarcinoma cell migration via activation of the MEK1/2 pathway. Oncol Lett. 2011;2:821-5. 
21. Borad MJ, Gores GJ, Roberts LR. Fibroblast growth factor receptor 2 fusions as a target for treating cholangiocarcinoma. Curr Opin Gastroenterol. 2015;31:264-8.

22. Jain A, Borad MJ, Kelley RK, et al. Cholangiocarcinoma with FGFR genetic aberrations: a unique clinical phenotype. $J$ CO Precision Oncology. 2018:2:1-12.

23. Borad MJ, Champion MD, Egan JB, et al. Integrated genomic characterization reveals novel, therapeutically relevant drug targets in FGFR and EGFR pathways in sporadic intrahepatic cholangiocarcinoma. PLOS Genet. 2014;10:e1004135.

24. Javle M, Lowery M, Shroff RT, et al. Phase II study of BGJ398 in patients with FGFR-altered advanced cholangiocarcinoma. J Clin Oncol. 2018;36:276-82.

25. Javle M, Kelley RK, Roychowdhury S, et al. AB051. P-19. A phase II study of infigratinib (BGJ398) in previously-treated advanced cholangiocarcinoma containing FGFR2 fusions. Hepatobiliary surg Nutr. 2019;8(Suppl. 1):AB051.

26. Goyal L, Saha SK, Liu LY, et al. Polyclonal secondary FGFR2 mutations drive acquired resistance to FGFR inhibition in patients with FGFR2 fusion-positive cholangiocarcinoma. Cancer Discov 2017;7:252-63.

27. Papadopoulos KP, El-Rayes BF, Tolcher AW, et al. A phase 1 study of ARQ 087, an oral pan-FGFR inhibitor in patients with advanced solid tumours. Br J Cancer. 2017:117:1592-9.

28. Mazzaferro V, El-Rayes BF, Cotsoglou C, et al. ARQ 087, an oral pan-fibroblast growth factor receptor (FGFR) inhibitor, in patients (pts) with advanced intrahepatic cholangiocarcinoma (iCCA) with FGFR2 genetic aberrations. J Clin Oncol. 2017;35(Suppl. 15):4017.

29. Goyal L, Arkenau H-T, Tran B, et al. Early clinical efficacy of TAS-120, a covalently bound FGFR inhibitor, in patients with cholangiocarcinoma. Ann Oncol. 2017;28 (Suppl. 3):iii145.

30. Meric-Bernstam F, Arkenau H, Tran B, et al. Efficacy of TAS-120, an irreversible fibroblast growth factor receptor (FGFR) inhibitor, in cholangiocarcinoma patients with FGFR pathway alterations who were previously treated with chemotherapy and other FGFR inhibitors. Ann Oncol. 2018;29(Suppl. 5):v100.

31. Vogel A, Sahai V, Hollebecque A, et al. FIGHT-202: A phase II study of pemigatinib in patients (pts) with previously treated locally advanced or metastatic cholangiocarcinoma (CCA) Ann Oncol. 2019:30(Suppl. 5):v876

32. Soria J-C, Strickler JH, Govindan R, et al. Safety and activity of the pan-fibroblast growth factor receptor (FGFR) inhibitor erdafitinib in phase 1 study patients (Pts) with molecularly selected advanced cholangiocarcinoma (CCA). I Clin Oncol. 2017 35(Suppl. 15):4074.

33. Park JO, Feng Y-H, Chen Y-Y, et al. Updated results of a phase lla study to evaluate the clinical efficacy and safety of erdafitinib in Asian advanced cholangiocarcinoma (CCA) patients with FGFR alterations. J Clin Oncol. 2019;37(Suppl. 15):4117.

34. Miyano SW, Arai Y, Matsui J, Shibata T. E7090, a novel and selective FGFR inhibitor, for the treatment of cholangiocarcinoma cells harboring FGFR2-fusion genes. Cancer Res. 2017;
77(Suppl. 13):2095.

35. Miyamoto M, Ojima H, Iwasaki M, et al. Prognostic significance of overexpression of C-Met oncoprotein in cholangiocarcinoma. Br J Cancer. 2011;105:131-8.

36. Labib PL, Goodchild G, Pereira SP. Molecular pathogenesis of cholangiocarcinoma. BMC Cancer. 2019;19:185

37. Sia D, Tovar V, Moeini A, Llovet JM. Intrahepatic cholangiocarcinoma: pathogenesis and rationale for molecula therapies. Oncogene. 2013;32:4861-70.

38. Wang SC WY, Lui TT, Weng SW, et al. Amplification and overexpression of the MET gene in intrahepatic cholangiocarcinoma correlate with adverse pathological features and worse clinical outcome. Int I Clin Exp Pathol. 2017:10:6809-17.

39. Lee J, Park SH, Chang HM, et al. Gemcitabine and oxaliplatin with or without erlotinib in advanced biliary-tract cancer: a multicentre, open-label, randomised, phase 3 study. Lancet Oncol. 2012;13:181-8

40. Malka D, Cervera P, Foulon S, et al. Gemcitabine and oxaliplatin with or without cetuximab in advanced biliary-tract cancer (BINGO): a randomised, open-label, non-comparative phase 2 trial. Lancet Oncol. 2014:15:819-28.

41. Leone F, Marino D, Cereda S, et al. Panitumumab in combination with gemcitabine and oxaliplatin does not prolong survival in wild-type KRAS advanced biliary tract cancer: A randomized phase 2 trial (Vecti-BIL study). Cancer. 2016;122:574-81.

42. Sohal DP, Mykulowycz K, Uehara T, et al. A phase II trial of gemcitabine, irinotecan and panitumumab in advanced cholangiocarcinoma. Ann Oncol. 2013;24:3061-5.

43. Kim RD, Poklepovic AS, Nixon AB, et al. Multi institutional phase II trial of single agent regorafenib in refractory advanced biliary cancers. J Clin Oncol. 2018:36(Suppl. 15):4082.

44. Demols A, Borbath I, Van Den Eynde M, et al. Regorafenib after failure of gemcitabine and platinum-based chemotherapy for locally advanced (nonresectable) and metastatic biliary tumors: A randomized double-blinded placebo-controlled phase II trial. J Clin Oncol. 2019;37(Suppl. 4):345.

45. Sun W, Patel A, Normolle D, et al. A phase 2 trial of regorafenib as a single agent in patients with chemotherapy-refractory, advanced, and metastatic biliary tract adenocarcinoma. Cancer. 2019;125:902-9.

46. Yi JH, Thongprasert S, Lee J, et al. A phase II study of sunitinib as a second-line treatment in advanced biliary tract carcinoma: a multicentre, multinational study. Eur J Cancer. 2012;48:196-201.

47. Lee JK, Capanu M, O'Reilly EM, et al. A phase II study of gemcitabine and cisplatin plus sorafenib in patients with advanced biliary adenocarcinomas. Br J Cancer. 2013;109:915-9.

48. Moehler M, Maderer A, Schimanski C, et al. Gemcitabine plus sorafenib versus gemcitabine alone in advanced biliary tract cancer: a double-blind placebo-controlled multicentre phase II AlO study with biomarker and serum programme. Eur J Cancer. 2014;50:3125-35.
49. Bengala C, Bertolini F, Malavasi N, et al. Sorafenib in patients with advanced biliary tract carcinoma: a phase II trial. Br J Cancer. 2010;102:68-72.

50. El-Khoueiry AB, Rankin CJ, Ben-Josef E, et al. SWOG 0514: a phase II study of sorafenib in patients with unresectable or metastatic gallbladder carcinoma and cholangiocarcinoma. Invest New Drugs. 2012;30:1646-51

51. El-Khoueiry AB, Rankin C, Siegel AB, et al. S0941: a phase 2 SWOG study of sorafenib and erlotinib in patients with advanced gallbladder carcinoma or cholangiocarcinoma. Br J Cancer. 2014;110:882-7.

52. Wainberg ZA, Lassen UN, Elez E, et al. Efficacy and safety of dabrafenib (D) and trametinib (T) in patients (pts) with BRAF V600E-mutated biliary tract cancer (BTC): A cohort of the ROAR basket trial. J Clin Oncol. 2019;37(Suppl. 4):187.

53. Javle M, Churi C, Kang HC, et al. HER2/neu-directed therapy for biliary tract cancer. J Hematol Oncol. 2015;8:58.

54. Javle MM, Murugesan K, Shroff RT, et al. Profiling of 3,634 cholangiocarcinomas (CCA) to identify genomic alterations (GA), tumor mutational burden (TMB), and genomic loss of heterozygosity (gLOH). J Clin Oncol. 2019;37(Suppl. 15):4087.

55. Ioka T, Ueno M, Oh D-Y, et al. Evaluation of safety and tolerability of durvalumab (D) with or without tremelimumab (T) in patients (pts) with biliary tract cancer (BTC). J Clin Oncol. 2019;37(Suppl. 4):387.

56. Bang $\mathrm{Y}-\mathrm{J}$, Ueno $\mathrm{M}$, Malka $\mathrm{D}$, et al. Pembrolizumab (pembro) for advanced biliary adenocarcinoma: Results from the KEYNOTE-02 (KN028) and KEYNOTE-158 (KN158) basket studies. J Clin Oncol. 2019;37(Suppl. 15):4079.

57. Kim RD, Kim DW, Alese $\mathrm{OB}$, et al. A phase II study of nivolumab in patients with advanced refractory biliary tract cancers (BTC). J Clin Oncol. 2019;37(Suppl. 15):4097.

58. Ueno M, Ikeda M, Morizane $\mathrm{C}$, et al. Nivolumab alone or in combination with cisplatin plus gemcitabine in Japanese patients with unresectable or recurrent biliary tract cancer: a non-randomised, multicentre, open-label, phase 1 study. Lancet Gastroenterol Hepatol. 2019;4:611-21.

59. Spizzo G, Puccini A, Xiu J, et al. Frequency of BRCA mutation in biliary tract cancer and its correlation with tumor mutational burden (TMB) and microsatellite instability (MSI). J Clin Oncol. 2019;37(Suppl. 15):4085.

60. Faraoni I, Graziani G. Role of BRCA mutations in cancer treatment with poly(ADP-ribose) polymerase (PARP) inhibitors. Cancers (Basel). 2018;10:487

61. Golan T, Raitses-Gurevich M, Kelley RK, et al. Overall survival and clinical characteristics of BRCA-associated cholangiocarcinoma: a multicenter retrospective study. Oncologist. 2017;22:804-10

62. Ross JS, Wang K, Gay L, et al. New routes to targeted therapy of intrahepatic cholangiocarcinomas revealed by next-generation sequencing. Oncologist. 2014;19:235-42. 